\title{
Targeting DNAJC19 overcomes tumor growth and lung metastasis in NSCLC by regulating PI3K/AKT signaling
}

Ji Zhou ${ }^{1 \dagger}$, Yang Peng ${ }^{2 \dagger}$, Ying-chun Gao ${ }^{3 \dagger}$, Tai-yu Chen ${ }^{4}$, Peng-cheng Li ${ }^{5}, K_{e} X^{5}$, Tao Liu ${ }^{5}$ and Tao Ren ${ }^{5^{*}}$ (1)

\begin{abstract}
Background: Some driver oncogenes are still unknown in non-small-cell lung cancer (NSCLC). DNAJC19, a major component of the translocation machinery of mitochondrial membranes, is a disease-associated protein. Herein, we report the role of DNAJC19 in NSCLC cell growth and metastasis.

Methods: Immunohistochemistry (IHC) was performed to investigate DNAJC19 expression in NSCLC clinical samples. For knockdown or overexpression assays in A549 or NCI-H1299 lung cancer cells, lentiviral vectors were used. After assessment of cell functions, DNAJC19-knockdown A549 cells were further applied to establish mouse xenograft and metastasis tumor models. Assessments based on the RNA-seq data, western blotting, PCR and IHC were performed for the mechanistic study.

Results: Expression of DNAJC19 was higher in tumors than in noncancerous adjacent tissues. Survival analysis indicated that low DNAJC19 levels were correlated with an increased progression-free survival rate. ShRNA-mediated knockdown of DNAJC19 markedly inhibited cell growth, viability, migration and invasion. Moreover, RNA-seq analysis revealed that the PI3K/AKT signaling pathway was involved in molecular events when A549 cells were treated with shDNAJC19. In addition, DNAJC19 knockdown decreased PI3Kp85a, AKT and p-AKT expression in A549 cells, and cellular functions were greatly rescued in DNAJC19-knockdown A549 cells by ectopic overexpression of AKT. Furthermore, tumor xenograft growth and lung metastasis were markedly repressed in the shDNAJC19 group compared to the control group. As expected, the expression levels of DNAJC19, PI3K and AKT in xenograft mouse samples were also lower in the shDNAJC19 group than in the shCtrl group.
\end{abstract}

Conclusions: DNAJC19 greatly promotes NSCLC cell growth and lung metastasis by regulating PI3K/AKT signaling, providing a novel therapeutic target for treating NSCLC patients.

Keywords: DNAJC19, Targeted therapy, AKT, Non-small-cell lung cancer, Metastasis

*Correspondence: rentao509@cmc.edu.cn

†ji Zhou, Yang Peng and Ying-chun Gao contributed equally to this work ${ }^{5}$ Oncology Department, Clinical Medical College and The First Affiliated Hospital of Chengdu Medical College, 278 Baoguang St, XinduDistr, Chengdu 610500, Sichuan, China

Full list of author information is available at the end of the article

\section{Background}

Non-small-cell lung cancer (NSCLC) has the highest cancer incidence worldwide [1,2]. With the adoption of sophisticated surgical and radiotherapeutic modalities, especially tyrosine kinase inhibitors (TKIs) [2, 3] and programmed death-1/programmed death-ligand 1 (PD-1/PD-L1) inhibitors [4-6], overall survival has been increased for NSCLC patients. However, compared to that in other cancer patients, the survival time in most NSCLC patients is relatively short because of

(c) The Author(s) 2021. This article is licensed under a Creative Commons Attribution 4.0 International License, which permits use, sharing, adaptation, distribution and reproduction in any medium or format, as long as you give appropriate credit to the original author(s) and the source, provide a link to the Creative Commons licence, and indicate if changes were made. The images or other third party material in this article are included in the article's Creative Commons licence, unless indicated otherwise in a credit line to the material. If material is not included in the article's Creative Commons licence and your intended use is not permitted by statutory regulation or exceeds the permitted use, you will need to obtain permission directly from the copyright holder. To view a copy of this licence, visit http://creativecommons.org/licenses/by/4.0/. The Creative Commons Public Domain Dedication waiver (http://creativecommons.org/publicdomain/zero/1.0/) applies to the data made available in this article, unless otherwise stated in a credit line to the data. 
adverse outcomes, such as metastasis and relapse [7]. A deeper understanding of the biological traits of NSCLC is required to improve the therapeutic efficacy against tumor cells.

DNAJC19 is a homologous protein of yeast Pam18/ Tim14 (yPam18), a component of the mitochondrial protein import machinery in the inner mitochondrial membrane, which is thought to be involved in the ATPdependent transport of transit proteins from the inner cell membrane to the mitochondrial matrix. It has been reported that DNAJC19 interacts with the mitochondrial prohibit in complex, which disrupts the functional integrity of mitochondria by disturbing phospholipid homeostasis, leading to mitochondrial cristae alterations. In addition, mutations in DNAJC19 have been linked to clinical manifestations, including failure to thrive, ataxia, dilated cardiomyopathy, cryptorchidism, and even hypospadias. Mitochondrial dysfunction, a common phenotypic characteristic of tumor cells, is thought to contribute to the development and progression of various cancers [8]. This raises the possibility that overexpression or mutation of DNAJC19 is associated with tumor development and progression. However, the oncogenic role of DNAJC19 and the associated underlying mechanisms in lung cancer are still far from elucidated.

Recently, the identification of additional driver oncogenes, such as epidermal growth factor receptor (EGFR), anaplastic lymphoma kinase (ALK), ROS proto-oncogene 1 (ROS1), and rearranged during transfection (RET), in NSCLC patients has led to new targeted treatments [9], greatly improving clinical practice. However, almost $8-40 \%$ of driver oncogenes are still unknown in NSCLC $[9,10]$.

Our data show that DNAJC19 is overexpressed in NSCLC tumor tissues compared to normal tissues. Moreover, despite no difference in overall survival (OS), NSCLC patients with high DNAJC19 expression have poorer progression-free survival (PFS). Furthermore, we used A549 and NCI-H1299 NSCLC cell lines in vitro to investigate the biofunctions of DNAJC19 in cell processes, including cell growth, proliferation, migration, invasion. Interestingly, we found that DNAJC19 increased tumor metastasis and affected xenograft tumor growth in a nude mouse model. More importantly, we explored the underlying regulatory mechanism and report for the first time that the DNAJC19/PI3K/AKT signaling pathway is involved in NSCLC cell proliferation and lung migration.

\section{Materials and methods}

Human specimens and cell culture

The collection and use of human samples and animal experiments were approved by the ethical review committees of the First Affiliated Hospital of Chengdu Medical College (No. 2020CYFYIRB-BA-89).

Human specimens were collected from 39 NSCLC patients during surgery at the First Affiliated Hospital, Chengdu Medical College. The patient characteristics are summarized in Table 1.

The human lung cancer cell lines A549, NCIH1299,Hela, and SiHa, were obtained from American Type Culture Collection (Manassas, VA, USA), and NCI-H1975, 95-D and MRC-5 were obtained from Cell Bank of the Chinese Academy of Sciences (Shanghai, People's Republic of China) and cultured in RPMI1640 medium (Corning, NY, USA) supplemented with $10 \%$ heat-inactivated FBS. The cells were maintained at $37^{\circ} \mathrm{C}$ in a $5 \% \mathrm{CO} 2 / 95 \%$ air humidified incubator.

\section{Cell transfection}

Cell transfections were performed using lentiviral vectors (Shanghai GeneChem Co., Ltd.). DNAJC19, AKT and MYC expression vectors and small hairpin RNAs (shRNAs) targeting DNAJC19 were purchased from GeneChem Co. (Shanghai, China). The DNAJC19 shRNA target sequence was as follows: TTTGCAGGC CGTTACGTTT, and the control sequence was TTC TCCGAACGTGTCACGT.

Table 1 Correlations between clinicopathological characteristics and DNAJC19 in NSCLC patients

\begin{tabular}{lcccc}
\hline Parameter & \multicolumn{2}{l}{$\begin{array}{l}\text { DNAJC19 } \\
\text { expression }\end{array}$} & $\mathbf{X}^{\mathbf{2}}$ & $\boldsymbol{P}$ \\
\cline { 2 - 3 } & Low & High & & \\
\hline Sex & 15 & 8 & 0.0307 & 0.8619 \\
$\quad$ Male & 10 & 6 & & \\
Female & & & & \\
Age & 9 & 7 & 0.7270 & 0.3939 \\
$<60$ & 16 & 7 & & \\
$\geq 60$ & & & & \\
Type & 16 & 9 & 0.0003 & 0.9858 \\
Adenocarcinoma & 9 & 5 & & \\
Squamous carcinoma & & & & \\
Staging & 18 & 10 & 0.0015 & 0.9697 \\
T1c-2b & 7 & 4 & & \\
T3-4 & 19 & 8 & 1.4980 & 0.2210 \\
N0 & 6 & 6 & & \\
N1-2 & & & & \\
Tissue & & & & \\
Carcinoma tissue & 25 & 14 & 4.4860 & 0.0342 \\
Paracarcinoma tissue & 18 & 2 & & \\
\hline
\end{tabular}




\section{Celigo assay, MTT assay and flow cytometric analysis}

The indicated cells were plated in a 96-well plate (Corning, NY, USA) at a density of 2,000 cells/well. After $24 \mathrm{~h}$ of cell seeding, Celigo (Nexcelom, Massachusetts, USA) was used to count the cell numbers daily for 5 days [11].

The indicated cells were plated in a 96-well plate at a density of 2,000 cells/well. MTT methods were used to assess cell viability at 24, 48, 72, 96 and $120 \mathrm{~h}$ after cell seeding (Shanghai Dimgguo, China) according to the manufacturer's instructions [11].

Apoptotic cells were detected using flow cytometric analysis with the Annexin V-APC kit (eBioscience, ThermoFisher, Shanghai, China) [12] according to the manufacturer's instructions.

\section{RNA extraction and qPCR}

Total RNA was extracted using TRIzol reagent (Shanghai Pufei Biotech Co., Ltd. Shanghai, China) according to the manufacturer's instructions. Reverse transcription (RT) and PCR were performed with a High-Capacity cDNA Reverse Transcription kit and a QuantiTect SYBR Green PCR kit (Qiagen, Shanghai, China), respectively. The primer sequences (Shanghai GeneChem Co., Ltd.) were as follows: DNAJC19 forward, 5'-ACAAAACGGGAA GCAGCATTA-3' and reverse, 5'-AGGAGATCCTCC TTTGTCAGG- $3^{\prime}$ and GAPDH forward, 5'-TGACTT CAACAGCGACACCCA-3' and reverse, 5'-CACCCT GTTGCTGTAGCCAAA-3'.

\section{Cell migration and invasion}

Cell migration was investigated by a Transwell $(8-\mu \mathrm{m}$ pore size; Corning, NY, USA) assay [13]. In brief, A549 and NCI-H1299 cells suspended in serum-free medium were seeded into the upper chambers, and the lower chambers were filled with RPMI-1640 supplemented with $30 \%$ FBS. After $16 \mathrm{~h}$ of incubation at $37^{\circ} \mathrm{C}$, the cells were fixed and stained.

And the invasion assays [14] were carried out via using Matriel (Corning, NY, USA), which was moved into the upper chambers and incubated $2 \mathrm{~h}$ in $37^{\circ} \mathrm{C}$ incubator. A549 and NCI-H1299 cells suspended in serum-free medium were seeded into the upper chambers, and the lower chambers were filled with RPMI-1640 supplemented with $30 \% \mathrm{FBS}$. After $30 \mathrm{~h}$ of incubation at $37^{\circ} \mathrm{C}$, the cells were fixed and stained.

The number of migrated cells was counted using an inverted microscope (CKX41, Olympus, Japan)(200X, 9 fields/chamber). And the assays were triplicate.

\section{Western blot and immunohistochemistry assays}

Western blotting and immunohistochemistry (IHC) were performed as previously described [15]. Antibodies against DNAJC19, PI3Kp85a, p-AKT(T308), AKT, GAPDH, CDH2, MMP2, TWIST, MYC, Caspase 3 and p-mTOR were purchased from Abcam (Cambridge, MA, USA. The catalog is ab230187, ab191606, ab8933, ab18785, ab9485, ab18203, ab37150, ab50887, ab32072, ab32351 and ab109268, respectively), and antibodies against CDH1, ERK1/2, p-P38, P38, MMP9, Snail, mTOR, NFkB-p65, p-ERK1/2, VIM, p-NFkB-p65, p- $\beta$ Catenin, $\beta$-Catenin, Slug were bought from CST (Massachusetts, USA. The catalog is 14472S, \#9107, \#4631, \#8690, 13667s, 3879s, \#2972, \#8242, \#4376, 3932s, 3033s, 2009s, 9562s, \#9585, respectively). And antibody against FN1 was purchased for R\&D (Minnesota, USA. The catalog is MAB19182). The antibodies against PARP1, Cytochrome $\mathrm{C}$ were bought from ZEN BIO (Chengdu, China. The catalog is $385279,200,758$, respectively).

\section{RNA-seq analysis}

RNA was extracted following the TRIzol reagent manual. RNA was precipitated in 1:1 isopropanol $(\mathrm{v} / \mathrm{v})$ and $1 \mu \mathrm{L}$ glycogen at $-20^{\circ} \mathrm{C}$ overnight. Sequencing libraries were generated using NEBNext ${ }^{\circledR}$ UltraTM RNA Library Prep Kit for Illumina ${ }^{\circledR}$ (New England BioLabs, Ltd., USA) following the manufacturer's recommendations [16, 17]. After adenylation of the 3' ends of the DNA fragments, the NEBNext Adaptor with a hairpin loop structure was ligated to the fragments to prepare for hybridization. To select cDNA fragments of preferentially $250-300 \mathrm{bp}$ in length, the library fragments were purified with AMPure XP system (Beckman Coulter, Beverly, USA). Then, $3 \mu \mathrm{L}$ USER Enzyme (NEB, USA) was used with size-selected, adaptor-ligated cDNA at $37{ }^{\circ} \mathrm{C}$ for 15 min followed by 5 min at $95{ }^{\circ} \mathrm{C}$ before PCR. PCR was performed with Phusion High-Fidelity DNA polymerase, Universal PCR primers and Index (X) Primer. The PCR products were purified (AMPure XP system), and library quality was assessed on the Agilent Bioanalyzer 2100 system.

The clustering of the index-coded samples was performed on a cBot Cluster Generation System using TruSeq PE Cluster Kit v3-cBot-HS (Illumia) according to the manufacturer's instructions. After cluster generation, the library preparations were sequenced on an Illumina Novaseq platform, and $150 \mathrm{bp}$ paired-end reads were generated. Gene Ontology (GO) and Kyoto Encyclopedia of Genes and Genomes (KEGG) pathway analyses were conducted by clusterProfiler (version 3.4.4).

\section{Animal experiments}

The animal experiments were approved by the local ethical review committees (No. 2020CYFYIRB-BA-89).

Animal experiments were conducted using 6-weekold female Balb/c nude mice [18]. A total of $1 \times 10^{7}$ DNAJC19-knockdown A549 cells or negative control 
A549 cells were suspended in $100 \mu$ of phosphatebuffered saline and subcutaneously injected into each mouse to establish the xenograft model; every group had 10 mice. After the mice were euthanized via using intraperitoneal of $2 \%$ sodium pentobarbital about $70 \mathrm{~s}$, xenograft tumors were dissected and then weighed. The samples were split, and one part was formalinfixed and paraffin-embedded, while the other was stored in liquid nitrogen.

For the tumor metastasis model [19], mice were injected via the tail vein with DNAJC19-knockdown or negative control A549 cells $\left(2 \times 10^{6}\right)(\mathrm{n}=5$ per group). After 32 days, an in vivo imaging system for small animals (Lumina LT, Perkin Elmer) was used to assess the metastatic tumors. The animals were anesthetized with isoflurane during the scanning period.

\section{Statistics}

The data are expressed as the mean \pm standard deviation (SD). One-way analysis of variance (ANOVA) followed by Tukey's multiple comparison procedure was used for comparisons of multiple groups. A value of $\mathrm{P}<0.05$ was considered to be statistically significant. The assays were performed at least three times independently.

\section{Results}

DNAJC19 is highly expressed in NSCLC tumor tissue

First, we analyzed the relationship between DNAJC19 expression and clinical characteristics in NSCLC patients using the MEXPRESS data base. DNAJC19 expression was higher in primary tumors than in normal tissues (Fig. 1A). IHC was performed to evaluate DNAJC19 expression in 39 NSCLC samples. As expected, compared to noncancerous adjacent tissue, cancer tissue showed significant overexpression of DNAJC19 (Fig. 1B and

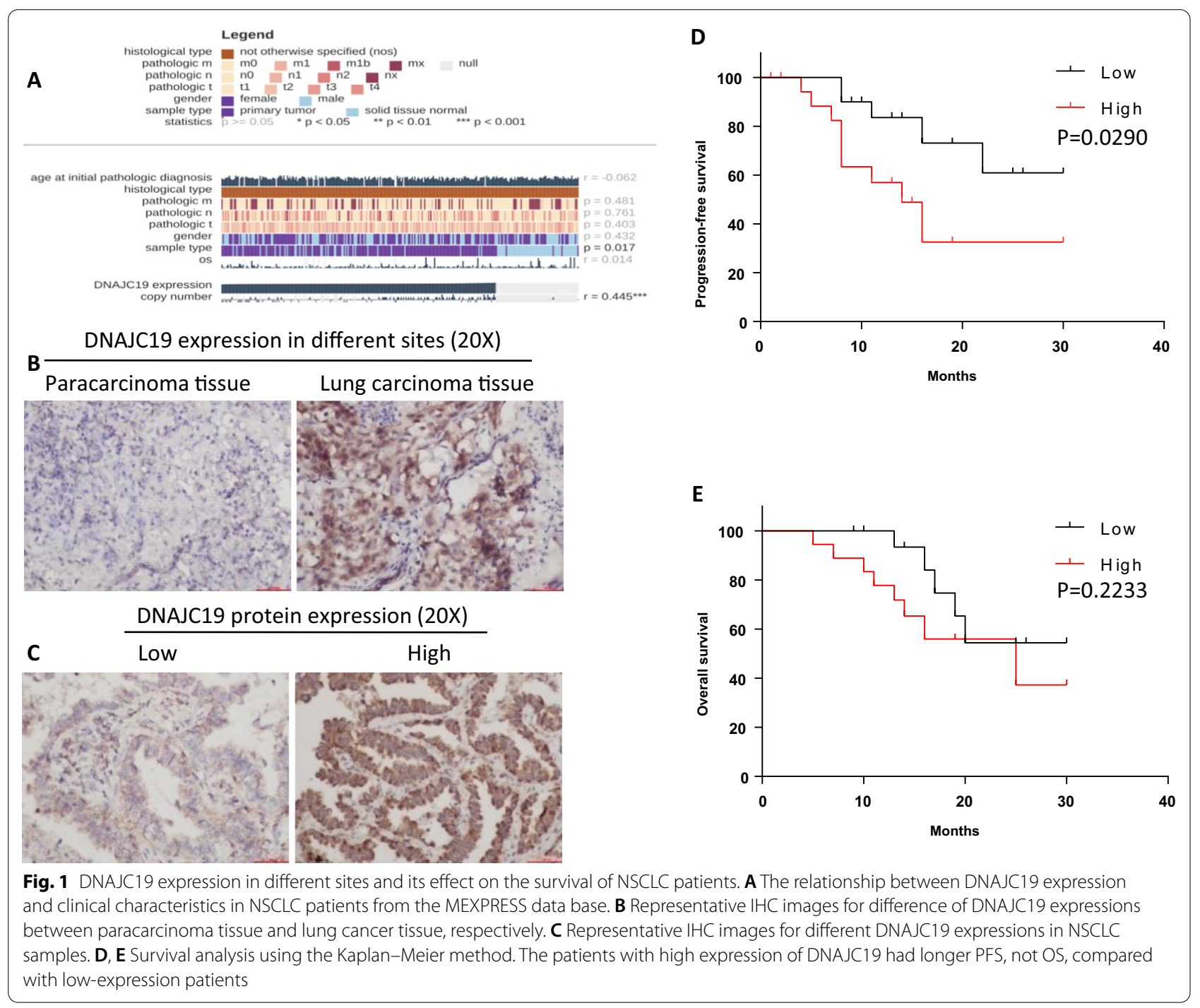


Table 1). In addition, the DNAJC19 expression level was diverse in different cancer cases (Fig. 1C). Next, we analyzed the correlation between DNAJC19 expression and clinicopathologic characteristics. As shown in Table 1, no significant differences, except for in the tissue category, were found between the low and high DNAJC19 expression groups in clinical parameters, including sex, age, T staging, $\mathrm{N}$ staging, and tumor type. Furthermore, we carried out survival analysis using the Kaplan-Meier method. A low level of DNAJC19 in NSCLC patients was correlated with increased progression-free survival but not overall survival. These data suggest that DNAJC19 expression in NSCLC patients is high and is associated with poor prognosis.

\section{Inhibition of DNAJC19 with shRNA decreases NSCLC cell growth, viability, and migration ability in vitro}

The clinical data indicated that DNAJC19 overexpression may be associated with poor prognosis in NSCLC patients. Therefore, we carried out in vitro experiments to investigate whether and how the DNAJC19 protein plays a role in lung cancer cells.

First, we assessed the protein expression of DNAJC19 in different lung cancer cells. As shown in Additional file 1: Figure S1A, the level of DNAJC19 was generally high in A549, 95-D, NCI-H1975 and NCI-H1299 cells. Among these, A549 cells had the lowest level, and NCIH1299 cells had a relatively higher level of DNAJC19 protein expression and were therefore used in subsequent assays.

We designed shRNAs targeting DNAJC19 (GenBank Accession No. NM_145261) through the online tool provided by BLOCK-iT RNAi Designer (http://rnaidesign er.lifetechnologies.com/rnaiexpress/design.do). The target sequence TTTGCAGGCCGTTACGTTT and control sequence TTCTCCGAACGTGTCACGT were used to design oligonucleotides, which were synthesized by Shanghai Generay Biotech Co., Ltd. After oligonucleotide annealing, we successfully constructed a recombinant lentiviral vector expressing shRNA against the human DNAJC19 gene (Lv-shRNA-DNAJC19) by using the linearized vector GV115 (Shanghai GeneChem Co., Ltd. Shanghai, China) (Additional file 1: Figure S1B-D). Next, we treated A549 and NCI-H1299 cells with DNAJC19 shRNA (shDNAJC19) or control shRNA (shCtrl) (Additional file 1: Figures S1E and Fig. 2A) to investigate cell growth, viability, and migration. As shown in Fig. 2B, A549 and NCI-H1299 cell growth in the shDNAJC19treated group was markedly inhibited compared with that in the shCtrl group at days 4 and 5, respectively, as determined by the Celigo assay. Compared with the shCtrl group, the shDNAJC19 group had markedly decreased A549 and NCI-H1299 cell viability at days 4 and 5, respectively, as demonstrated by the MTT assay (Fig. 2C). We also found that A549 and NCI-H1299 cell migration and invasion were largely impeded in the shDNAJC19 group compared with the shCtrl group based on migration and invasion assays (Fig. 2D-G). The numbers of migrating cells in the shCtrl and shDNAJC19 groups were $144.3 \pm 4.0$ vs. $61.7 \pm 3.1$, respectively, for A549 cells and $235.7 \pm 4.5$ vs. $115.3 \pm 3.5$, respectively, for NCIH1299 cells (Fig. 2D, E). The numbers of invading cells in the shCtrl and shDNAJC19 groups were $256.3 \pm 4.0$ vs. $148.0 \pm 3.5$, respectively, for A549 cells and 177.4 \pm 4.0 vs $51.0 \pm 3.0$, respectively, for NCI-H1299 cells (Fig. 2F, G). The Annexin V-APC assay demonstrated that apoptosis of A549 and NCI-H1299 cells was clearly increased in the shDNAJC19 group compared with the shCtrl group (Fig. 2H). And we further used Western blotting to investigate the expression of apoptotic proteins from A549 cells treated with shDNAJC19, as shown in Fig. 2I, either caspase3 or parp1 was largely increased in the shDNAJC19 group compared with the shCtrl group; and the cytochrome $\mathrm{C}$ protein was decreased in mitochondria but increased in cytoplasm. Taken together, these results suggest that shRNA-mediated inhibition of DNAJC19 impairs cell growth, viability, migration and invasion in lung cancer cell lines.

\section{PI3K/AKT is involved in molecular events of DNAJC19 shRNA treatment based on RNA-seq analysis}

From the RNA-seq data of Fig. 5, there were 761 upregulated and 746 downregulated proteins based on an analysis of the RNA transcriptomes of 3 A549 samples treated with shDNAJC19 and 3 controls (Fig. 3A). Unsupervised hierarchical clustering distinguished shDNAJC19 from the control with minor overlap (Fig. 3B). The top enriched KEGG pathways were protein processing in endoplasmic, PI3K/AKT signaling pathway, MAPK signaling pathway, and microRNAs in cancer (Fig. 3C). These findings suggested that PI3K/AKT signaling is involved in the molecular events in A549 cells treated with shDNAJC19.

\section{DNAJC19 directly regulates PI3K/AKT signaling}

Next, we performed a western blot assay to investigate a panel of related proteins, including $\mathrm{m}$-TOR, MMP2, CDH2, p38, NFkB-P65, catenin, ERK1/2, MYC, PI3Kp85a, AKT and pAKT(T308), in A549 cells. Interestingly, we found that the protein levels of PI3K, AKT and p-AKT were markedly decreased in A549 cells treated with shDNAJC19 (Fig. 4A). PI3K/AKT signaling plays a key role in tumor cell growth, viability, proliferation, migration and invasion [20]. Next, we overexpressed AKT (Lv-AKT) in A549 lung cancer cells treated with DNAJC19 shRNA (Fig. 4B). As shown in Fig. $4 \mathrm{C}$, the Celigo assay revealed that the growth ability 


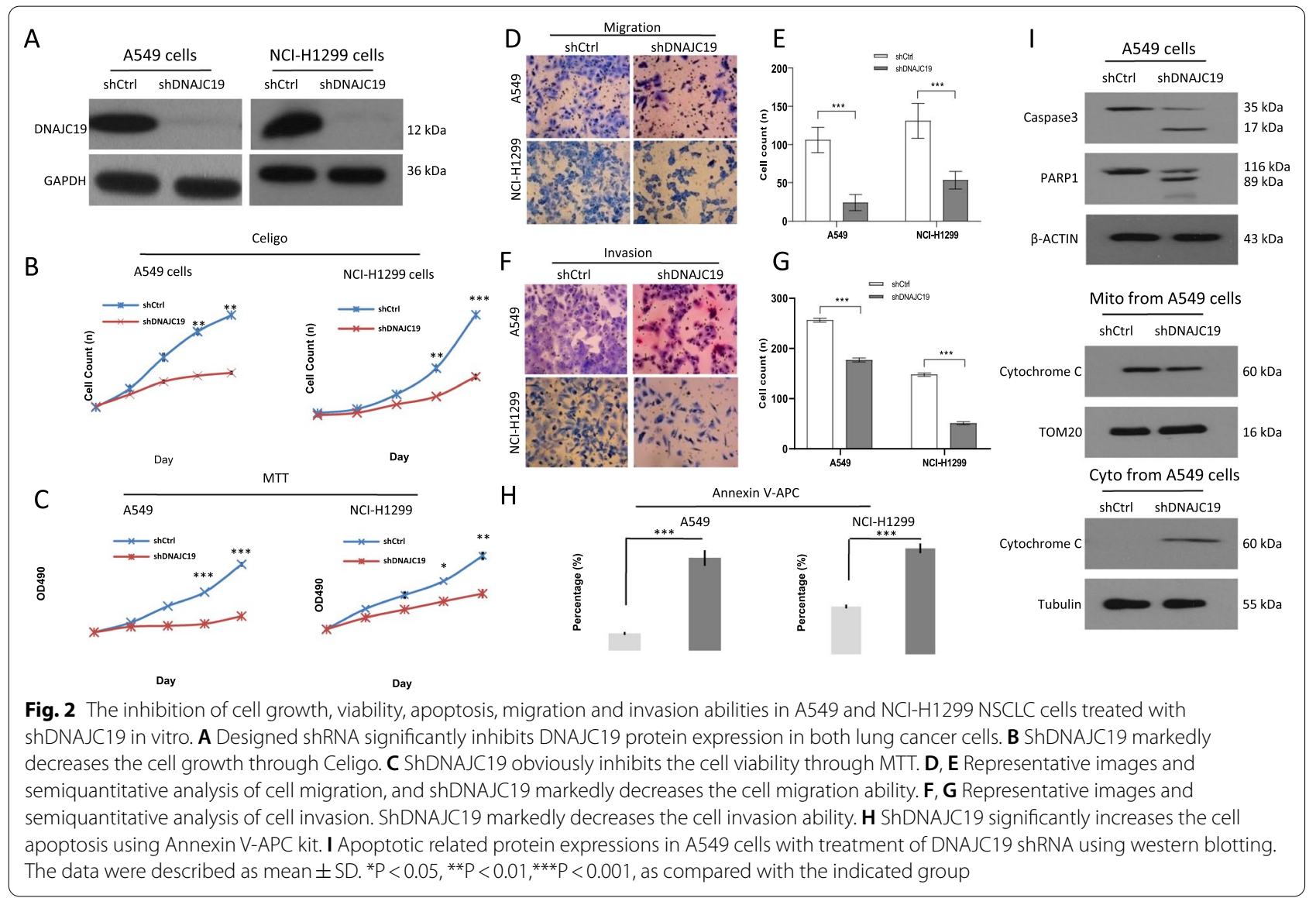

of DNAJC19 shRNA-treated A549 cells compared with DNAJC19 shRNA-knockdown cells was largely restored when $\mathrm{Lv}$-AKT was administered. These data suggest that AKT is regulated by DNAJC19 in lung cancer cells. We thus further tested cell viability by the MTT assay and migration of A549 cells treated with DNAJC19 shRNA. As expected, A549 cell viability was visibly enhanced in the DNAJC19 shRNA plus Lv-AKT group compared with the DNAJC19 shRNA-only group (Fig. 4D). The migration ability of A549 cells was also substantially rescued and further promoted in the combination group of shDNAJC19 plus Lv-AKT compared with the shDNAJC19-only group (Fig. 4E, F). Taken together, these results suggest that DNAJC19 regulates A549 cells probably through modulating PI3K/AKT.

\section{DNAJC19 has a positive role in xenograft tumor growth and tumor lung metastasis}

We further estimated the value of DNAJC19 as a potential antitumor target. We used A549 cells treated with Lv-shRNA-DNAJC19 or Lv-shRNA-Control to establish an animal model. In the xenograft mouse model, compared with the negative control Lv-shRNA-Control group (shCtrl group), the Lv-shRNA-DNAJC19 group (shDNAJC19 group) had markedly dampened tumor xenograft growth (Fig. 5A), both in terms of tumor weight $(\mathrm{P}<0.001)$ (Fig. $5 \mathrm{~B})$ and tumor volume at days 21,25 , and $27(\mathrm{P}<0.001)$ and at days 23 and $29(\mathrm{P}<0.0001)$ (Fig. 5C). Moreover, we investigated DNAJC19, PI3K and AKT in xenograft samples by IHC. As shown in Fig. 5F, DNAJC19, PI3K and AKT expression levels were lower in the shDNAJC19 group than in the shCtrl group (Fig. 5D). In the tumor metastasis model, fewer average metastatic tumor lesions in each mouse and fewer mice $(80 \%, 4 / 5)$ with lung metastasis were observed in the shDNAJC19 group than in the shCtrl group (Fig. 5E, F). Furthermore, we did HE staining to investigate whether the metastasis lesion exist in mice model. As shown in Fig. 5G, in lung tissue we saw the definitely intrapulmonary metastasis lesion in shControl group. Furthermore, we did immunohistochemistry to assess the $\mathrm{Ki}-67$ expression of tumor metastasis samples. Accordingly, ki-67 expressions are strongly high in shControl group than in shDNAJC19 group (Fig. $5 \mathrm{H}$ ). These results suggest that DNAJC19 facilitates xenograft tumor growth and tumor 

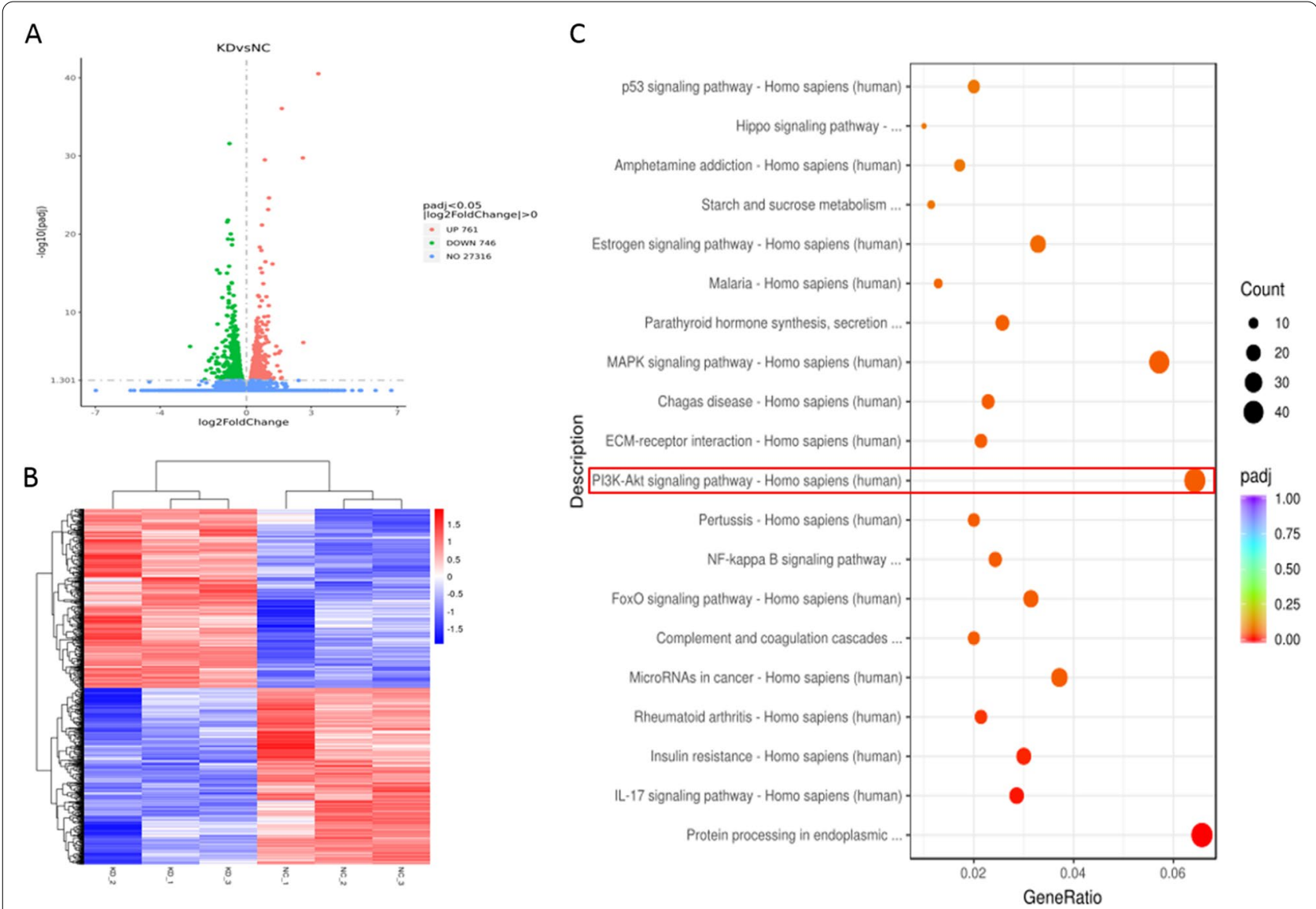

Fig. 3 RNA-seq data from A549 cells treated with shDNAJC19 or shRNA control. A Volcano plot of mRNA expression in A549 cells treated with shDNAJC19 or shCtrl. B Heatmap for each sample. C Bubble plot of top 20 enrichment pathways, including the PI3K/AKT pathway (Marked as the red frame)

metastasis by regulating PI3K/AKT signaling and is a new potential anticancer target molecule in NSCLC.

\section{Discussion}

Patients with advanced NSCLC have a poor prognosis and adverse outcomes, including recurrence and metastasis $[21,22]$. With the development of tyrosine kinase inhibitors (TKIs) [23, 24] and immune checkpoint inhibitors (ICIs), the clinical outcomes of advanced NSCLC patients have improved considerably. However, most of these patients develop primary or acquired drug resistance, indicating that many driver oncogenes or biological behaviors of NSCLC tumor cells are not fully understood and need to be further characterized. Overall, exploring novel driver oncogenes is urgently needed to overcome drug resistance and to identify potential therapeutic targets in NSCLC.

The protein encoded by the DNAJC19 gene is considered to participate in a complex involved in the ATP-dependent transport of transit peptide-containing proteins from the inner cell membrane to the mitochondrial matrix. DNAJC19, also named translocase of the inner mitochondrial membrane 14 (TIMM14), is responsible for maintaining the integrity of mitochondria and is linked to cardiovascular disease, as well as cancers [25]. Mitochondrial dysfunction due to changes in DNAJC19 is a common phenotype of tumor cells and is supposed to contribute to the initiation, development and progression of many solid cancers [8]. To our knowledge, we are the first to report the protumor property of DNAJC19 and its regulatory mechanisms in NSCLC.

In this study, we found that DNAJC19 was overexpressed in NSCLC carcinoma tissues, which is consistent with a MEXPRESS analysis from the TCGA data base. Further survival analysis indicated that NSCLC patients with enhanced DNAJC19 expression had poor progression-free survival. These data suggest that DNAJC19 may play an important role in NSCLC tumor development and progression. However, the function of DNAJC19 in NSCLC cells was not clear. 

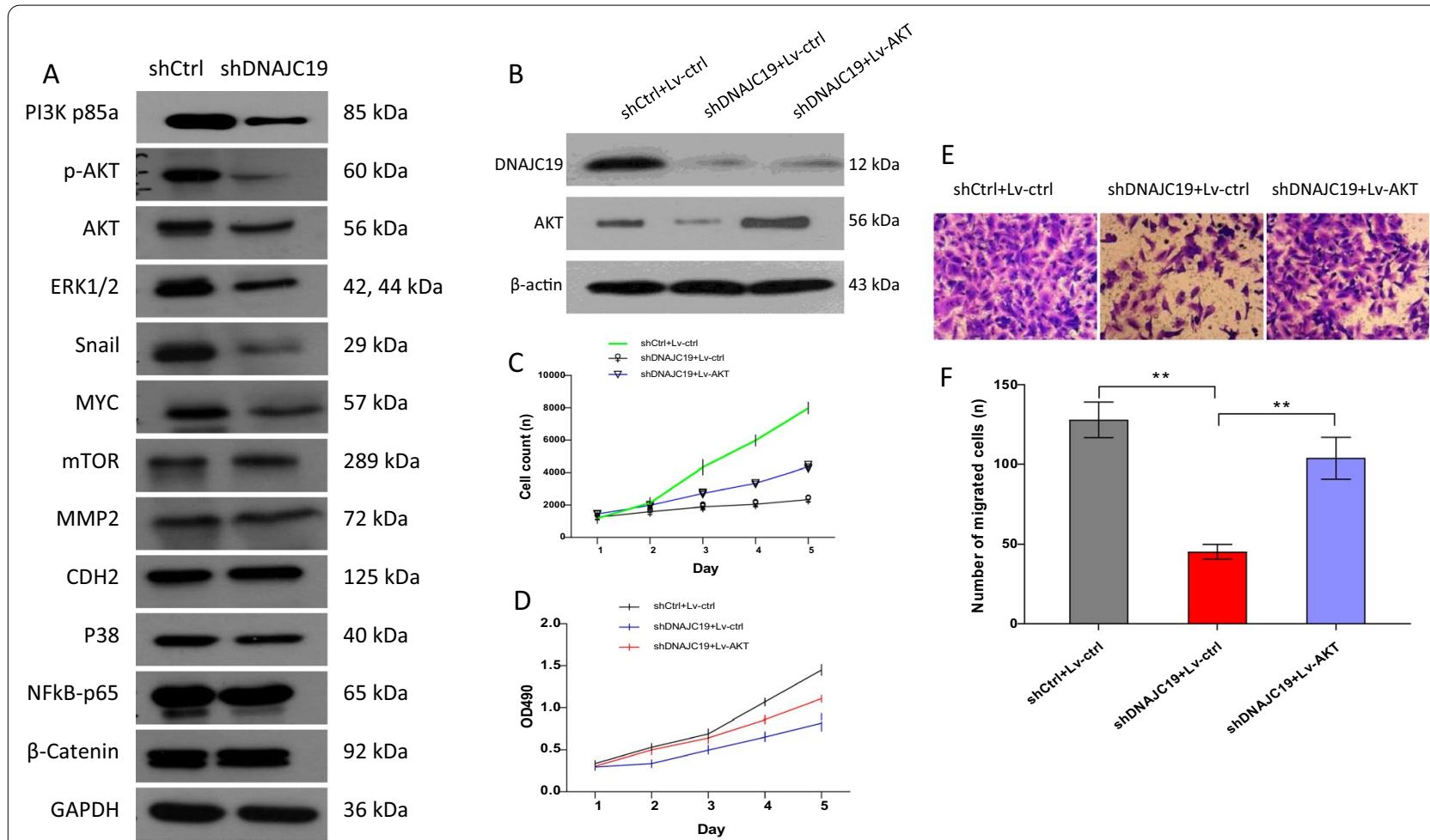

$\mathrm{F}$

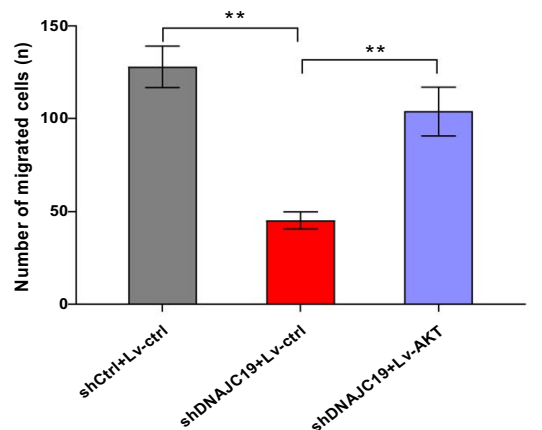

Fig. 4 PI3K/AKT pathway is regulated by DNAJC19. A The related proteins were investigated in A549 cells treated with shDNAJC19 or shRNA control through western blotting. $\mathbf{B}$ Lentiviral-mediated AKT (Lv-AKT) overexpression in A549 cells with treatment of DNAJC19 shRNA using western blotting. C The growth was strongly restored by overexpression of AKT in A549 cells concurrently treated with shDNAJC19 via Celigo assay. D The cell viability was rescued by AKT overexpression in A549 cells with the concurrent treatment with shDNAJC19 using MTT assay. E, F The migration ability of A549 cells was strongly enhanced by simultaneous AKT overexpression and DNAJC19 knockdown. Data are shown as mean \pm SD. **P $<0.01$, when in contrast with the indicated group

Aberrant regulation of DNAJC19 induces mitochondrial dysfunction, which is critical for cell survival [26]. First, we successfully designed shRNA to knock down DNAJC19 in A549 and NCI-H1299 NSCLC cells and found that DNAJC19 knockdown markedly inhibited NSCLC cell growth and metastasis. Notably, RNA-seq analysis verified that the PI3K/AKT signaling pathway was involved in molecular events when A549 cells were treated with shDNAJC19. Moreover, suppression of DNAJC19 also decreased the expression of PI3K, and AKT proteins in A549 cells. Interestingly, the cell growth, viability and migration abilities were rescued in DNAJC19-knockdown A549 cells by overexpressing AKT. Interestingly, the change of MYC, ERK $1 / 2$ or Snail was obvious in A549 cells treated with shDNAJC19 as shown in Fig. 4A, so we speculate that PI3K/AKT/MYC [27], PI3K/AKT/ERK [28] and/or PI3K/AKT/Snail [29] pathway is responsible for DNAJC19 mediated proliferation and migration. We will further research the underlying regulatory mechanism of DNAJC19 in NSCLC in future. Well known, PI3K/AKT signaling is crucial in the regulation of cellular growth and metabolism [30].
In NSCLC, the PI3K/AKT pathway has been implicated in both tumorigenesis and the progression of disease, and somatic mutations of PIK3CA and amplifications of PIK3CA are frequently found in patients with NSCLC [31]. Moreover, cancer cell-intrinsic pathways including PI3K mediate immunosuppression in lung cancer [32]. $\mathrm{AKT}$ is involved in tumor cell proliferation in breast cancer [33], stem cell properties and apoptosis in non-smallcell lung cancer [34] and tumor progression in gastric cancer [35]. Furthermore, the mouse xenograft model showed that tumor size and weight were significantly decreased in the DNAJC19-knockdown group compared with the negative control group. As expected, compared with the shCtrl group, the shDNAJC19 group exhibited fewer lung metastatic lesions. In addition, the expression levels of DNAJC19, PI3K and AKT in xenograft mouse samples were lower in the shDNAJC19 group than in the shCtrl group. These data suggested that PI3K/AKT is an previously unidentified target of DNAJC19.

In brief, we found for the first time that shRNAmediated repression of DNAJC19 greatly attenuated tumor cell growth and metastasis by regulating PI3K/ 


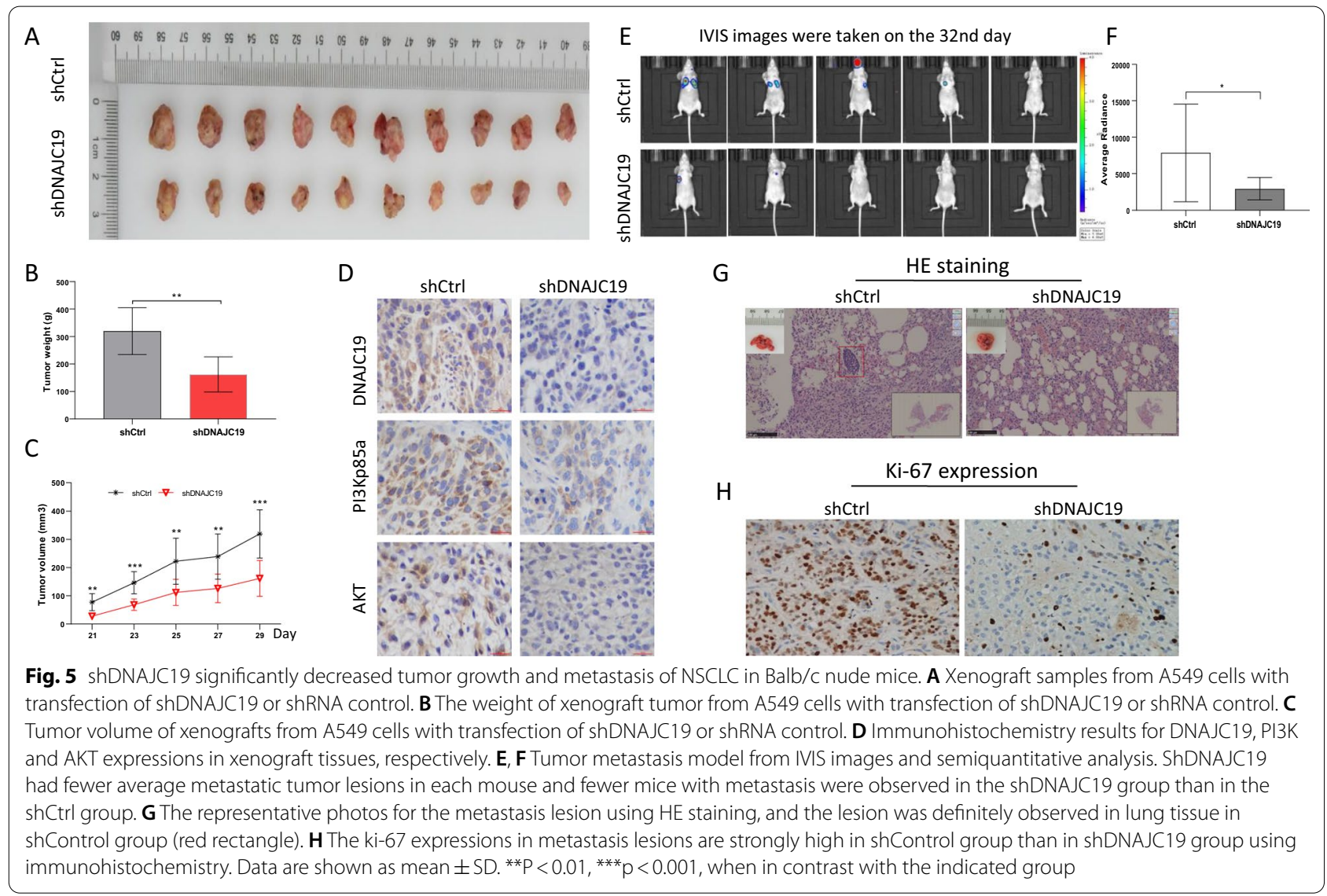

AKT signaling, providing a novel therapeutic target for NSCLC patients.

\section{Conclusion}

We reported that high expression of DNAJC19 protein was associated with poor prognosis in NSCLC patients. We successfully designed shRNA to especially and patently inhibit DNAJC19 expression and functions. Finally, we found for the first time that shRNA-mediated repression of DNAJC19 greatly attenuated tumor cell growth and intrapulmonary metastasis by regulating PI3K/AKT, highlighting DNAJC19 as a novel therapeutic target for treating NSCLC patients.

\section{Abbreviations}

NSCLC: Non-small-cell lung cancer; FBS: Fetal bovine serum; IHC: Immunohistochemistry; TKIs: Tyrosine kinase inhibitors; ICls: Immune checkpoint inhibitors; PD-1/PD-L1: Programmed death-1/programmed death-ligand 1; EGFR: Epidermal growth factor receptor; ALK: Anaplastic lymphoma kinase; ROS1: ROS proto-oncogene 1; OS: Overall survival; PFS: Progression-free survival; shRNAs: Small hairpin RNAs.

\section{Supplementary Information}

The online version contains supplementary material available at https://doi. org/10.1186/s12935-021-02054-z.
Additional file 1: Figure S1. The designed shRNA sequence successfully inhibited the protein and mRNA levels of DNAJC19 in A549 and $\mathrm{NCl}-\mathrm{H} 1299$ cells. A. The protein expressions of DNAJC19 in different NSCLC cells. B, C Constructed recombinant lentiviral vector expressing shRNA against human DNAJC19 gene (Lv-shRNA DNAJC19) by using the linearized vector GV115. D. The cell status after infection with shDNAJC19 or shCtrl. E. The mRNA level of DNAJC19 measured in lung cancer cells treated with shDNAJC19 or shControl by qPCR.

\section{Acknowledgements}

We thank AJE for improving the language and data interpretation of the manuscript.

\section{Authors' contributions}

RT and ZJ conceived the idea, designed the experiments, and wrote the manuscript with input from all the coauthors. PY, YCG, CTY, LPC, YF, ZYG, and ZJ resigned shRNA of DNAJC19 and performed the in vitro cellular and in vivo animal experiments, including collection of clinical data. XK and LT performed MEXPRESS analysis and survival analysis. All authors approved the final version. All authors read and approved the final manuscript.

\section{Funding}

The collection, analysis, and interpretation of data in this study were supported by grants from the applied basic research program, Science \& Technology Department of Sichuan Province (2020YJ0451), Introduction Foundation of High-level Talents of The First Affiliated Hospital, Chengdu Medical College (CYFY-GQ22), The Special Project of the First Affiliated Hospital, Chengdu Medical College (CYFY2019ZD03), and The Cultivation Project of Key Subjects of the First Affiliated Hospital, Chengdu Medical College. The sponsor reviewed and approved the study protocol and the final version of the manuscript. All 
the analytic decisions were made by the authors, and the final version of the manuscript was approved by all authors.

\section{Availability of data and materials}

The datasets used in the current study are available from the corresponding author on reasonable request.

\section{Declarations}

\section{Ethics approval and consent to participate}

This study was approved by the Ethics Committee of The First Affiliated Hospital of Chengdu Medical College (the Approval Number is 2020CYFYIRB-BA-89).

\section{Consent to publish}

Not applicable.

\section{Competing interests}

The authors declare no competing interests.

\begin{abstract}
Author details
${ }^{1}$ Health Management Centre, Clinical Medical College and The First Affiliated Hospital of Chengdu Medical College, 278 Baoguang St, Xindu Distr Chengdu 610500, Sichuan, China. ${ }^{2}$ Hematology Department, Clinical Medical College and The First Affiliated Hospital of Chengdu Medical College, Chengdu 610500, China. ${ }^{3}$ Oncology Department, Pengzhou People's Hospital, Chengdu 611900, China. ${ }^{4}$ Clinical Medical College of Chengdu Medical College, Chengdu 610500, China. ${ }^{5}$ Oncology Department, Clinical Medical College and The First Affiliated Hospital of Chengdu Medical College, 278 Baoguang St, XinduDistr, Chengdu 610500, Sichuan, China.
\end{abstract}

Received: 19 February 2021 Accepted: 25 June 2021

Published online: 03 July 2021

\section{References}

1. Siegel RL, Miller KD, Jemal A. Cancer statistics. CA Cancer J Clin. 2019;69(2019):7-34.

2. Oronsky B, Ma P, Reid TR, Cabrales P, Lybeck M, Oronsky A, Oronsky N, Carter CA. Navigating the "No Man's Land" of TKI-failed EGFR-mutated non-small cell lung cancer (NSCLC): a review. Neoplasia. 2018;20:92-8.

3. Soria JC, Ohe Y, Vansteenkiste J, Reungwetwattana T, Chewaskulyong B, Lee KH, Dechaphunkul A, Imamura F, Nogami N, Kurata T, Okamoto I, Zhou C, Cho BC, Cheng Y, Cho EK, Voon PJ, Planchard D, Su WC, Gray JE, Lee SM, Hodge R, Marotti M, Rukazenkov Y, Ramalingam SS, Investigators F. Osimertinib in untreated EGFR-mutated advanced non-small-cell lung cancer. N Engl J Med. 2018;378:113-25.

4. Ledford $\mathrm{H}$, Else $\mathrm{H}$, Warren M. Cancer immunologists scoop medicine Nobel prize. Nature. 2018;562:20-1.

5. Liu C, Zheng S, Jin R, Wang X, Wang F, Zang R, Xu H, Lu Z, Huang J, Le Y, Mao S, Wang Y, Feng X, Sun N, Wang Y, He J. The superior efficacy of anti-PD-1/PD-L1 immunotherapy in KRAS-mutant non-small cell lung cancer that correlates with an inflammatory phenotype and increased immunogenicity. Cancer Lett. 2020;470:95-105.

6. Rizvi H, Sanchez-Vega F, La K, Chatila W, Jonsson P, Halpenny D, Plodkowski A, Long N, Sauter JL, Rekhtman N, Hollmann T, Schalper KA, Gainor JF, Shen R, Ni A, Arbour KC, Merghoub T, Wolchok J, Snyder A, Chaft JE, Kris MG, Rudin CM, Socci ND, Berger MF, Taylor BS, Zehir A, Solit DB, Arcila ME, Ladanyi M, Riely GJ, Schultz N, Hellmann MD. Molecular determinants of response to anti-programmed cell death (PD)-1 and anti-programmed death-ligand 1 (PD-L1) blockade in patients with non-small-cell lung cancer profiled with targeted next-generation sequencing. J Clin Oncol. 2018;36:633-41.

7. Ferrara R, Mezquita L, Texier M, Lahmar J, Audigier-Valette C, Tessonnier L, Mazieres J, Zalcman G, Brosseau S, Le Moulec S, Leroy L, Duchemann B, Lefebvre C, Veillon R, Westeel V, Koscielny S, Champiat S, Ferte C, Planchard D, Remon J, Boucher ME, Gazzah A, Adam J, Bria E, Tortora G, Soria JC, Besse B, Caramella C. Hyperprogressive disease in patients with advanced non-small cell lung cancer treated with PD-1/PD-L1 inhibitors or with single-agent chemotherapy. JAMA Oncol. 2018;4:1543-52.

8. Heinemeyer T, Stemmet M, Bardien S, Neethling A. Underappreciated roles of the translocase of the outer and inner mitochondrial membrane protein complexes in human disease. DNA Cell Biol. 2019;38:23-40.

9. Skoulidis F, Heymach JV. Co-occurring genomic alterations in non-smallcell lung cancer biology and therapy. Nat Rev Cancer. 2019;19:495-509.

10. Chan BA, Hughes BG. Targeted therapy for non-small cell lung cancer: current standards and the promise of the future. Transl Lung Cancer Res. 2015:4:36-54.

11. Aarestrup FM, Albeyatti A, Armitage WJ, Auffray C, Augello L, Balling R, Benhabiles N, Bertolini G, Bjaalie JG, Black M, Blomberg N, Bogaert P, Bubak M, Claerhout B, Clarke L, De Meulder B, D’Errico G, Di Meglio A, Forgo N, Gans-Combe C, Gray AE, Gut I, Gyllenberg A, Hemmrich-Stanisak G, Hjorth L, loannidis Y, Jarmalaite S, Kel A, Kherif F, Korbel JO, Larue C, Laszlo M, Maas A, Magalhaes L, Manneh-Vangramberen I, Morley-Fletcher E, Ohmann C, Oksvold P, Oxtoby NP, Perseil I, Pezoulas V, Riess O, Riper H, Roca J, Rosenstiel P, Sabatier P, Sanz F, Tayeb M, Thomassen G, Van Bussel J, Van den Bulcke M, Van Oyen H. Towards a European health research and innovation cloud (HRIC). Genome Med. 2020;12:18.

12. Ma JT, Zhang XY, Cao R, Sun L, Jing W, Zhao JZ, Zhang SL, Huang LT, Han CB. Effects of Dynamin-related protein 1 regulated mitochondrial dynamic changes on invasion and metastasis of lung cancer cells. J Cancer. 2019;10:4045-53.

13. Johnson FM, Saigal B, Talpaz M, Donato NJ. Dasatinib (BMS-354825) tyrosine kinase inhibitor suppresses invasion and induces cell cycle arrest and apoptosis of head and neck squamous cell carcinoma and non-small cell lung cancer cells. Clin Cancer Res. 2005;11:6924-32.

14. Kim YJ, Kim JH, Kim O, Ahn EJ, Oh SJ, Akanda MR, Oh IJ, Jung S, Kim KK, Lee JH, Kim HS, Kim H, Lee KH, Moon KS. Caveolin-1 enhances brain metastasis of non-small cell lung cancer, potentially in association with the epithelial-mesenchymal transition marker SNAIL. Cancer Cell Int. 2019;19:171.

15. Pan ST, Zhou J, Yang F, Zhou SF, Ren T. Proteomics reveals a therapeutic vulnerability via the combined blockade of APE1 and autophagy in lung cancer A549 cells. BMC Cancer. 2020;20:634

16. Xing S, Zeng T, Xue N, He Y, Lai YZ, Li HL, Huang Q, Chen SL, Liu WL. Development and validation of tumor-educated blood platelets integrin alpha 2b (ITGA2B) RNA for diagnosis and prognosis of non-small-cell lung cancer through RNA-seq. Int J Biol Sci. 2019;15:1977-92.

17. Park YS, Kim S, Park DG, Kim DH, Yoon KW, Shin W, Han K. Comparison of library construction kits for mRNA sequencing in the Illumina platform. Genes Genomics. 2019;41:1233-40.

18. Miwa T, Kanda M, Umeda S, Tanaka H, Shimizu D, Tanaka C, Kobayashi D, Hayashi M, Yamada S, Nakayama G, Koike M, Kodera Y. Establishment of peritoneal and hepatic metastasis mouse xenograft models using gastric cancer cell lines. In Vivo. 2019;33:1785-92.

19. Gan L, Meng J, Xu M, Liu M, Qi Y, Tan C, Wang Y, Zhang P, Weng W, Sheng W, Huang $M$, Wang Z. Extracellular matrix protein 1 promotes cell metastasis and glucose metabolism by inducing integrin beta4/FAK/SOX2/HIF1alpha signaling pathway in gastric cancer. Oncogene. 2018;37:744-55.

20. Hinz N, Jucker M. Distinct functions of AKT isoforms in breast cancer: a comprehensive review. Cell Commun Signal. 2019;17:154.

21. Kloten V, Lampignano R, Krahn T, Schlange T. Circulating tumor cell PD-L1 expression as biomarker for therapeutic efficacy of immune checkpoint inhibition in NSCLC. Cells. 2019. https://doi.org/10.1155/2016/3460462.

22. Recondo G, Facchinetti F, Olaussen KA, Besse B, Friboulet L. Making the first move in EGFR-driven or ALK-driven NSCLC: first-generation or nextgeneration TKI? Nat Rev Clin Oncol. 2018;15:694-708.

23. MokTS, Cheng Y, Zhou X, Lee KH, Nakagawa $K$, Niho S, Lee M, Linke $R$, Rosell R, Corral J, Migliorino MR, Pluzanski A, Sbar El, Wang T, White JL, Wu $\mathrm{YL}$. Improvement in overall survival in a randomized study that compared dacomitinib with gefitinib in patients with advanced non-small-cell lung cancer and EGFR-activating mutations. J Clin Oncol. 2018:36:2244-50.

24. Ramalingam SS, Vansteenkiste J, Planchard D, Cho BC, Gray JE, Ohe Y, Zhou C, Reungwetwattana T, Cheng Y, Chewaskulyong B, Shah R, Cobo M, Lee KH, Cheema P, Tiseo M, John T, Lin MC, Imamura F, Kurata T, Todd A, Hodge R, Saggese M, Rukazenkov Y, Soria JC. Overall survival with osimertinib in untreated, EGFR-mutated advanced NSCLC. N Engl J Med. 2020;382:41-50. 
25. Sinha D, Srivastava S, D'Silva P. Functional diversity of human mitochondrial j-proteins is independent of their association with the inner membrane presequence translocase. J Biol Chem. 2016;291:17345-59.

26. Richter-Dennerlein R, Korwitz A, Haag M, Tatsuta T, Dargazanli S, Baker M, Decker T, Lamkemeyer T, Rugarli El, Langer T. DNAJC19, a mitochondrial cochaperone associated with cardiomyopathy, forms a complex with prohibitins to regulate cardiolipin remodeling. Cell Metab. 2014;20:158-71.

27. Wei C, Dong X, Lu H, Tong F, Chen L, Zhang R, Dong J, Hu Y, Wu G, Dong $X$. LPCAT1 promotes brain metastasis of lung adenocarcinoma by upregulating PI3K/AKT/MYC pathway. J Exp Clin Cancer Res. 2019;38:95.

28. Wang YJ, Lin JF, Cheng LH, Chang WT, Kao YH, Chang MM, Wang BJ, Cheng HC. Pterostilbene prevents AKT-ERK axis-mediated polymerization of surface fibronectin on suspended lung cancer cells independently of apoptosis and suppresses metastasis. J Hematol Oncol. 2017;10:72.

29. Wang Z, Li J, Long X, Jiao L, Zhou M, Wu K. MRPS16 facilitates tumor progression via the PI3K/AKT/snail signaling axis. J Cancer. 2020;11:2032-43.

30. Tan AC. Targeting the PI3K/Akt/mTOR pathway in non-small cell lung cancer (NSCLC). Thorac Cancer. 2020;11:511-8.

31. Scheffler M, Bos M, Gardizi M, Konig K, Michels S, Fassunke J, Heydt C, Kunstlinger H, Ihle M, Ueckeroth F, Albus K, Serke M, Gerigk U, Schulte W, Topelt K, Nogova L, Zander T, Engel-Riedel W, Stoelben E, Ko YD, Randerath W, Kaminsky B, Panse J, Becker C, Hellmich M, Merkelbach-Bruse S,
Heukamp LC, Buttner R, Wolf J. PIK3CA mutations in non-small cell lung cancer (NSCLC): genetic heterogeneity, prognostic impact and incidence of prior malignancies. Oncotarget. 2015;6:1315-26.

32. Altorki NK, Markowitz GJ, Gao D, Port JL, Saxena A, Stiles B, McGraw T, Mittal $V$. The lung microenvironment: an important regulator of tumour growth and metastasis. Nat Rev Cancer. 2019;19:9-31.

33. Riggio M, Perrone MC, Polo ML, Rodriguez MJ, May M, Abba M, Lanari C, Novaro V. AKT1 and AKT2 isoforms play distinct roles during breast cancer progression through the regulation of specific downstream proteins. Sci Rep. 2017;7:44244

34. Wang M, Wang X, Li Y, Xiao Q, Cui XH, Xiao GD, Wang JC, Xu CW, Ren H, Liu D. Nutlin-3-induced sensitization of non-small cell lung cancer stem cells to axitinib-induced apoptosis through repression of Akt1/Wnt signaling. Oncol Res. 2019;27:987-95.

35. Al-Teneiji A, Siriwardena K, George K, Mital S, Mercimek-Mahmutoglu S. Progressive cerebellar atrophy and a novel homozygous pathogenic DNAJC19 variant as a cause of dilated cardiomyopathy ataxia syndrome. Pediatr Neurol. 2016;62:58-61.

\section{Publisher's Note}

Springer Nature remains neutral with regard to jurisdictional claims in published maps and institutional affiliations.
Ready to submit your research? Choose BMC and benefit from:

- fast, convenient online submission

- thorough peer review by experienced researchers in your field

- rapid publication on acceptance

- support for research data, including large and complex data types

- gold Open Access which fosters wider collaboration and increased citations

- maximum visibility for your research: over $100 \mathrm{M}$ website views per year

At BMC, research is always in progress.

Learn more biomedcentral.com/submissions 